\title{
The study of Higgs boson decaying into tau tau in CMS
}

\author{
Michal BLUJ*i \\ LLR Ecole Polytechnique, France \\ E-mail: michal.bluj@cern.ch
}

In this note we review results of the search for the standard model Higgs boson in the tau tau decay channel at the CMS experiment. The analysed pp collision data correspond to an integrated luminosity of $4.9 \mathrm{fb}^{-1}$ and $19.4 \mathrm{fb}^{-1}$ collected at $\sqrt{s}=7 \mathrm{TeV}$ and $\sqrt{s}=8 \mathrm{TeV}$, respectively. An excess of events is observed over a broad range of Higgs mass hypotheses, with a maximum local significance of 2.93 standard deviations at $\mathrm{m}_{\mathrm{H}}=120 \mathrm{GeV}$, and compatible with the presence of the standard model Higgs boson of mass $125 \mathrm{GeV}$.

PACS: $14.80 . \mathrm{Bn}$

The European Physical Society Conference on High Energy Physics

18-24 July, 2013

Stockholm, Sweden

\footnotetext{
* Speaker.

$\dagger$ On behalf of the CMS Collaboration

${ }^{\ddagger}$ Also at National Centre for Nuclear Research, Poland
} 


\section{Introduction}

After the discovery of a new standard-model-like Higgs boson announced by the ATLAS and CMS Collaborations in July 2012 [1, 2] it is essential to determine the nature of this new boson. Observation the decay to tau pairs is an important ingredient of this program.

In this note are presented recent results of the search for the Higgs boson decaying to a tau lepton pair [3] preformed with $4.9 \mathrm{fb}^{-1}$ and $19.4 \mathrm{fb}^{-1}$ of pp collision data collected by the CMS experiment [4] at $\sqrt{s}=7 \mathrm{TeV}$ and $\sqrt{s}=8 \mathrm{TeV}$, respectively.

The $\mathrm{H} \rightarrow \tau \tau$ analysis significantly profits from a particle-flow algorithm $[5,6,7]$ which combines the information from all CMS subdetectors to identify and reconstruct in the optimal way the individual particles: charged hadrons, neutral hadrons, photons, muons, and electrons. These particles are then used to reconstruct the missing transverse energy $\left(E_{\mathrm{T}}^{\mathrm{miss}}\right)$, jets, hadronic tau decays $\left(\tau_{\mathrm{h}}\right)$, and to define the isolation of leptons.

\section{Analysis strategy}

In the search for the Higgs boson decay into to a tau lepton pair five independent $\tau$-pair final states are considered (ordered by their sensitivity): $\mu \tau_{\mathrm{h}}, \mathrm{e} \tau_{\mathrm{h}}, \mathrm{e} \mu, \tau_{\mathrm{h}} \tau_{\mathrm{h}}$, and $\mu \mu$, where $\tau_{\mathrm{h}}$ stands for tau decaying to hadrons. Events within each final state are categorised basing on the number of jets in an event having $p_{\mathrm{T}}>30 \mathrm{GeV}$ in order to enhance an impact of specific Higgs boson production processes, which leads to a better sensitivity of the analysis. A two-jet category is designed to select events produced in the vector boson fusion (VBF) production mode, which is achieved by requiring that the two jets are well separated in pseudorapidity with $\Delta \eta_{j j}>3.5$ and have invariant mass $\mathrm{m}_{j j}>500 \mathrm{GeV}$, and by a veto on additional central jets. A one-jet category is mainly sensitive on high- $p_{\mathrm{T}}$ Higgs boson produced in the gluon fusion process associated with a jet. Finally, a zerojet category being dominated by background is used only to better constrain analysis uncertainties on background normalisation, identification efficiencies, and energy scales. The one and zero-jet categories are subdivided basing on $p_{\mathrm{T}}$ of $\tau_{\mathrm{h}}$ ( $\mu$ for the $\mathrm{e} \mu$ and $\mu \mu$ channels) to exploit the high mass of the Higgs boson. In the specific case of the $\tau_{\mathrm{h}} \tau_{\mathrm{h}}$ final state the zero-jet category is not used and the one-jet one is not subdivided due to the jet and high- $p_{\mathrm{T}}$ of $\tau_{\mathrm{h}}$ requirements present already in a trigger definition. In addition, in the $\tau_{\mathrm{h}} \tau_{\mathrm{h}}$ final state it is required that $p_{\mathrm{T}}$ of the $\tau_{\mathrm{h}} \tau_{\mathrm{h}}$-plusmissing-transverse-energy system is bigger than $140 \mathrm{GeV}$ and $110 \mathrm{GeV}$ at the one-jet and the VBF categories, respectively.

Although an exact background composition depends on final state and event category it is typically dominated by irreducible background, with real tau leptons, from the Drell-Yan Z/ $\gamma^{\star} \rightarrow \tau \tau$ production, followed by reducible contributions, with jet misidentified as isolated tau, from the $\mathrm{W}(\ell v)+\mathrm{jets}, \mathrm{Z} / \gamma^{\star} \rightarrow \ell \ell, \mathrm{QCD}$ multijet and diboson production processes. Background is estimated mainly from the data. The dominant $\mathrm{Z} / \gamma^{*} \rightarrow \tau \tau$ contribution is estimated with a so-called embedding technique where reconstructed muons from real $\mathrm{Z} / \gamma^{*} \rightarrow \mu \mu$ events are replaced by simulated tau decays forming hybrid $\mathrm{Z} / \gamma^{*} \rightarrow \tau \tau$ events with an underlying activity from data. Background from $\mathrm{W}+$ jets production is modelled with simulated events normalised in a $\mathrm{W}+\mathrm{jets}$ dominated control region with the high-transverse mass of the lepton and missing transverse energy system. The QCD multijet background is estimated from same-sign events exploring the fact that charge of two 
misidentified leptons is uncorrelated. Finally, other small background components are estimated using simulated events normalised to predicted rates.

The variable sensitive to presence of the Higgs boson used to extract the signal contribution is an estimate of full ditau mass, $\mathrm{m}_{\tau \tau}$. It is calculated using a likelihood based method taking as input the kinematics of visible decay products and $E_{\mathrm{T}}^{\text {miss }}$. It gives better separation between $\mathrm{H}$ and $\mathrm{Z}$ than the mass of the visible ditau system. The combined distribution of the full ditau mass in all categories of the $\mu \tau_{\mathrm{h}}, \mathrm{e} \tau_{\mathrm{h}}$, e $\mu$, and $\tau_{\mathrm{h}} \tau_{\mathrm{h}}$ channels with each category weighted by the expected signal-to-background ratio in the mass range containing $68 \%$ of the signal is shown in Fig. 1.

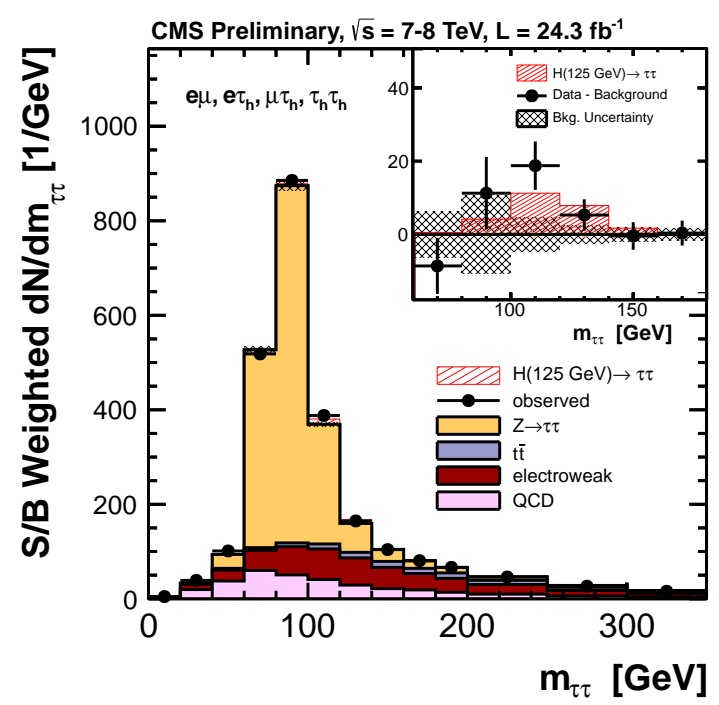

Figure 1: The combined distribution of the ditau mass in all categories of the $\mu \tau_{\mathrm{h}}$, e $\tau_{\mathrm{h}}$, e $\mu$, and $\tau_{\mathrm{h}} \tau_{\mathrm{h}}$ channels with each category weighted by the expected signal-to-background ratio. In the insert: The difference between the observed data and expected background distributions relative to the expected signal distribution for a standard model Higgs signal with $\mathrm{m}_{\mathrm{H}}=125 \mathrm{GeV}$.

Finally, the main $\mathrm{H} \rightarrow \tau \tau$ analysis discussed in this note is combined with the (low sensitivity) search for the Higgs boson decaying into a tau pair and produced in association with a $\mathrm{W}$ or $\mathrm{Z}$ boson, $\mathrm{V}(\mathrm{H} \rightarrow \tau \tau)$, described in Ref. [8].

\section{Results}

To search for the presence of the $\mathrm{H} \rightarrow \tau \tau$ signal a statistical procedure based on a profilelikelihood ratio test statistic is used [9]. The $\mathrm{m}_{\tau \tau}$ distributions, for each category of the five channels at $7 \mathrm{TeV}$ and $8 \mathrm{TeV}$, are used to build a binned likelihood function, involving expected background and Higgs boson signal scaled by a signal strength parameter $\mu=\sigma / \sigma_{\mathrm{SM}}$. The systematic uncertainties are represented by nuisance parameters.

The observed and expected 95\% confidence level, CL, limits on the Higgs boson cross section are given in Fig. 2a. A broad excess is found in the observed limit when compared to the background-only prediction. On the contrary, the observed limit is in a good agreement with expectation for the background plus signal of a standard model Higgs boson with $\mathrm{m}_{\mathrm{H}}=125 \mathrm{GeV}$, as 
shown in Fig. 2b. The significance of this excess is presented in Fig 3a. It reaches maximum of 2.93 standard deviations, $\sigma$, for $\mathrm{m}_{\mathrm{H}}=120 \mathrm{GeV}$, while at $\mathrm{m}_{\mathrm{H}}=125 \mathrm{GeV}$ it amounts to $2.85 \sigma$ compared to an expectation of $2.62 \sigma$ for the presence of a standard model Higgs boson. The measured signal strength yields $\mu=1.1 \pm 0.4$ at $125 \mathrm{GeV}$ (Fig. 3b).

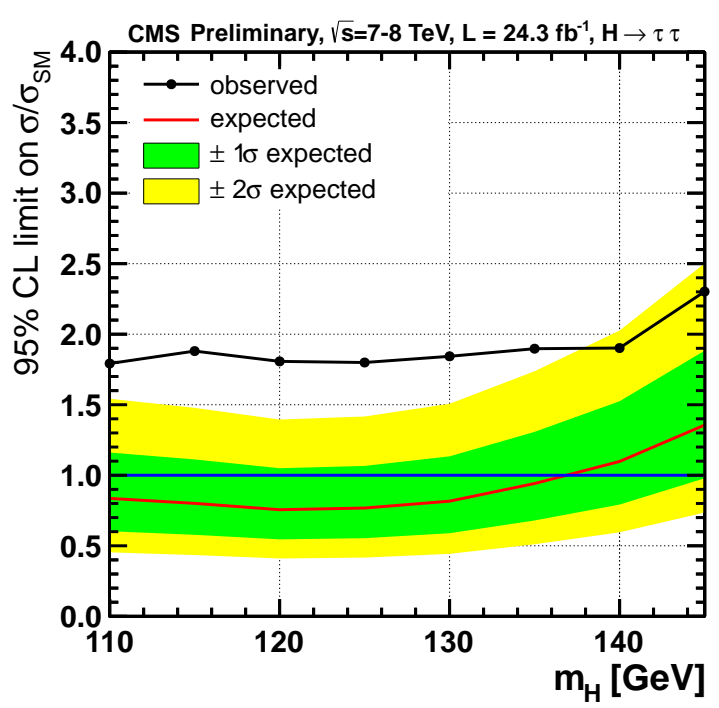

(a)

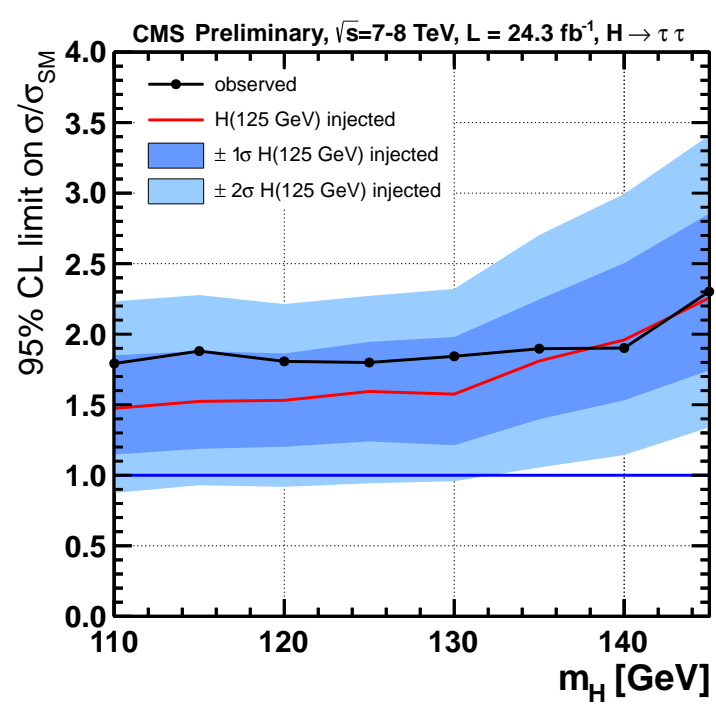

(b)

Figure 2: Observed 95\% CL upper limit on the signal cross section compared to the expected limit obtained in the background-only (a), and background plus signal of a standard model Higgs boson with $\mathrm{m}_{\mathrm{H}}=125 \mathrm{GeV}$ (b) hypotheses.

The $\mathrm{H} \rightarrow \tau \tau$ channel was also used to measure the mass of the Higgs boson to be $\mathrm{m}_{\mathrm{H}}=120_{-7}^{+9} \mathrm{GeV}$, in a good agreement with the value of $\mathrm{m}_{\mathrm{H}}=125.7 \pm 0.8 \mathrm{GeV}$ measured with the high-resolution $\mathrm{H} \rightarrow \mathrm{ZZ} \rightarrow 4 \ell$ and $\mathrm{H} \rightarrow \gamma \gamma$ channels [10].

\section{Summary}

A search for the Higgs boson in tau decay at CMS has been presented. It was performed in five different final states, $\mu \tau_{\mathrm{h}}, \mathrm{e} \tau_{\mathrm{h}}, \mathrm{e} \mu, \tau_{\mathrm{h}} \tau_{\mathrm{h}}$, and $\mu \mu$. The result is combined with the result of a search for the standard model Higgs boson decaying to tau pairs and produced in association with a vector boson. It is observed an excess of events above expected background giving a hint of the signal with significance of $2.85 \sigma(2.62 \sigma$ expected $)$ for $\mathrm{m}_{\mathrm{H}}=125 \mathrm{GeV}$ and signal strength $\mu=1.1 \pm 0.4$. A combination of the $\mathrm{H} \rightarrow \tau \tau$ and $\mathrm{V}(\mathrm{H} \rightarrow \mathrm{b} \overline{\mathrm{b}})[11]$ analyses leads to an evidence that the Higgs boson couples to down-type, third generation fermions with significance of $3.4 \sigma$ (3.4 $\sigma$ expected) [10].

\section{References}

[1] G. Aad et al. [ATLAS Collaboration], Observation of a new particle in the search for the Standard Model Higgs boson with the ATLAS detector at the LHC, Phys. Lett. B 716 (2012) 1

[arXiv:1207.7214 [hep-ex]]. 


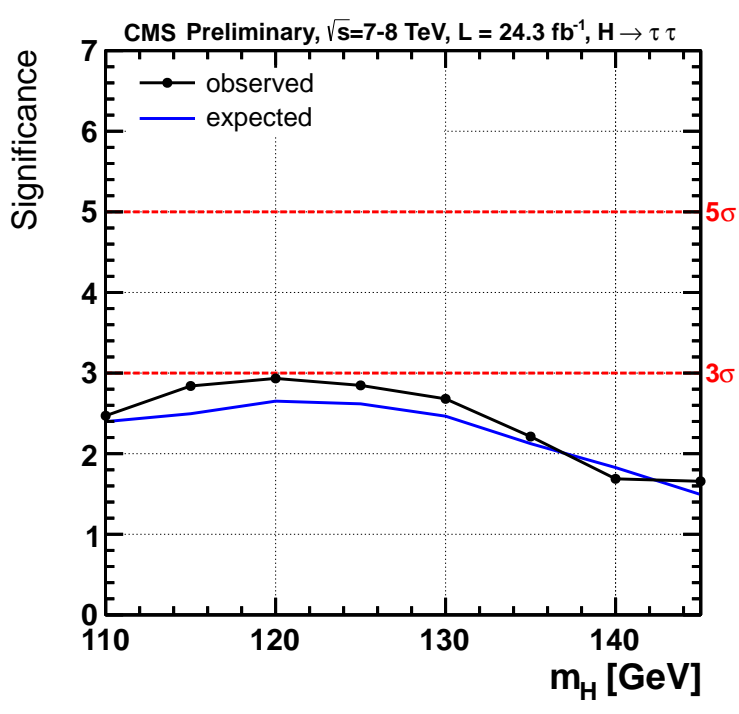

(a)

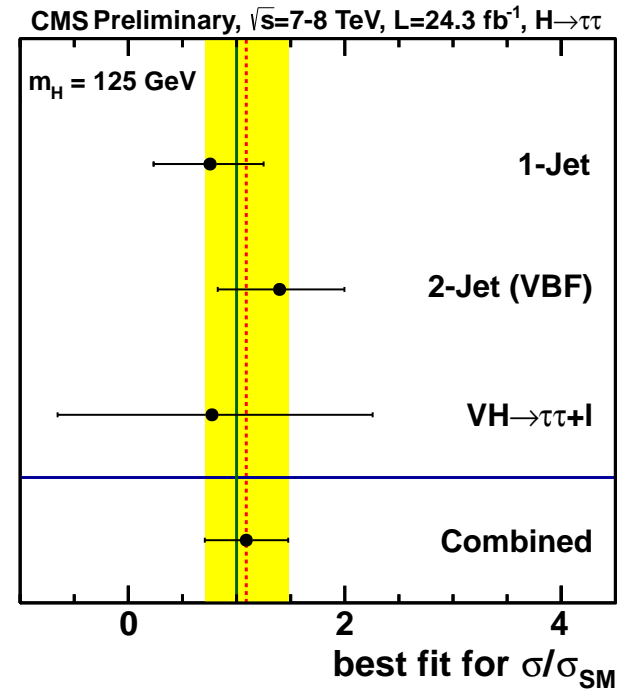

(b)

Figure 3: (a): Observed and expected significance as a function of $m_{H}$. (b): Best-fit signal strength, $\mu=\sigma / \sigma_{\mathrm{SM}}$, at $\mathrm{m}_{\mathrm{H}}=125 \mathrm{GeV}$ compared to the standard model expectation.

[2] S. Chatrchyan et al. [CMS Collaboration], Observation of a new boson at a mass of $125 \mathrm{GeV}$ with the CMS experiment at the LHC, Phys. Lett. B 716 (2012) 30 [arXiv:1207.7235 [hep-ex] ].

[3] CMS Collaboration, Search for the Standard-Model Higgs boson decaying to tau pairs in proton-proton collisions at sqrt(s) = 7 and 8 TeV, CMS PAS HIG-13-004 (2013)

[4] S. Chatrchyan et al. [CMS Collaboration], The CMS experiment at the CERN LHC, JINST 3 (2008) S08004.

[5] CMS Collaboration, Particl-Flow Event Reconstruction in CMS and Performance for Jets, Taus, and $E_{T}^{m i s s}$, CMS PAS PFT-09-001 (2009)

[6] CMS Collaboration, Commissioning of the Particle-flow Event Reconstruction in Minimum-Bias and Jet Events from pp Collisions at 7 TeV, CMS PAS PFT-10-002 (2010)

[7] CMS Collaboration, Commissioning of the particle-flow event reconstruction with leptons from $J / \psi$ and $W$ decays at $7 \mathrm{TeV}$, CMS PAS PFT-10-003 (2010)

[8] CMS Collaboration, Search for the standard model Higgs boson decaying to tau pairs produced in association with a W or Z boson, CMS PAS HIG-12-053 (2012)

[9] ATLAS and CMS Collaborations, LHC Higgs Combination Group, Procedure for the LHC Higgs boson search combination in Summer 2011, Technical Report, ATL-PHYS-PUB 2011-11, CMS NOTE 2011/005, (2011).

[10] CMS Collaboration, Combination of standard model Higgs boson searches and measurements of the properties of the new boson with a mass near 125 GeV, CMS-PAS-HIG-13-005 (2013)

[11] S. Chatrchyan et al. [ CMS Collaboration], Search for the standard model Higgs boson produced in association with a Wor a Z boson and decaying to bottom quarks, arXiv: 1310.3687 [hep-ex]. 